\title{
The Role of Quantitative Pharmacology in an Academic Translational Research Environment
}

\author{
Jeffrey S. Barrett ${ }^{1,2,3,4}$
}

Received 8 October 2007; accepted 21 November 2007; published online 5 February 2008

\begin{abstract}
Translational research is generally described as the application of basic science discoveries to the treatment or prevention of disease or injury. Its value is usually determined based on the likelihood that exploratory or developmental research can yield effective therapies. While the pharmaceutical industry has evolved into a highly specialized sector engaged in translational research, the academic medical research community has similarly embraced this paradigm largely through the motivation of the National Institute of Health (NIH) via its Roadmap initiative. The Clinical and Translational Science Award (CTSA) has created opportunities for institutions which can provide the multidisciplinary environment required to engage such research. A key component of the CTSA and an element of both the NIH Roadmap and the FDA Critical Path is the bridging of bench and bedside science via quantitative pharmacologic relationships. The infrastructure of the University of Pennsylvania/Children's Hospital of Philadelphia CTSA is highlighted relative to both research and educational objectives reliant upon quantitative pharmacology. A case study, NIH-sponsored research program exploring NK1r antagonism for the treatment NeuroAIDS is used to illustrate the application of quantitative pharmacology in a translational research paradigm.
\end{abstract}

KEY WORDS: Model-based drug development; NIH Roadmap; Quantitative pharmacology; Translational research.

\section{INTRODUCTION}

Academic research is an important component in the discovery and development of new molecular entities. Like their industrial and regulatory colleagues, academic scientists are engaged in responding to a changing $\mathrm{R} \& \mathrm{D}$ landscape which demands both efficiency and innovation. The concept of translational research has evolved over the past decade and has likewise been defined in many ways. A reasonable definition for translational research would be the application of basic scientific discoveries into clinically germane findings and, simultaneously, the generation of scientific questions based on clinical observations (1). A more lucid understanding can be found in the utilization of translational research methodologies in specific therapeutic areas such as oncology $(2,3)$, nephrology (4), pain management (5), and cognitive decline with aging (6). The historical and parallel context to be considered when

\footnotetext{
${ }^{1}$ Laboratory for Applied PK/PD, Clinical Pharmacology \& Therapeutics Division, The Children's Hospital of Philadelphia, Philadelphia, Pennsylvania, USA.

${ }^{2}$ Pediatrics Department, School of Medicine, University of Pennsylvania, Philadelphia, Pennsylvania, USA.

${ }^{3}$ Abramson Research Center, The Children's Hospital of Philadelphia, Rm 916H, 3615 Civic Center Blvd, Philadelphia, Pennsylvania 19104, USA.

${ }^{4}$ To whom correspondence should be addressed. (e-mail: barrettj@ email.chop.edu)
}

discussing the genesis of translational research is that while big and small Pharmaceutical Manufacturers Association (PhRMA) companies were contending with diminishing pipelines despite improvements in high throughput screening and other discovery-based innovations, academic medical centers were at a loss for how to connect the basic sciences in the post-genomic era to clinical researchers who had heard of the "bench" but had seldom visited. At the same time, their governing regulatory counterparts crafted the critical path (7) and roadmap (8) initiatives from the Food and Drug Administration (FDA) and National Institute of Health (NIH) respectively to address these concerns and provide suggestions for resolution. At the core of these proposals is a clear request for the creation of interdisciplinary teams that will define quantitative relationships bridging discovery and developmental science and challenging hypotheses regarding basic and clinical pharmacology, facilitating novel experimental designs and aiding in drug development decision making in general- "quantitative pharmacology" for lack of a better name.

\section{NIH ROADMAP AND THE CLINICAL AND TRANSLATION SCIENCE AWARD (CTSA)}

Academic research is funded by a variety of mechanisms including the public and private sectors with the major investment coming from the NIH which will use much of its projected \$28.6 billion 2007 budget to fund biomedical research in the United States (9). The structure of the NIH 
is composed primarily of disease-specific institutions which has historically created a compartmentalized focus on disease research; counter to new paradigms which encourage multidisciplinary teams and the basic-to-applied research continuum in general. The NIH Roadmap initiative (http://nihroadmap.nih. gov) seeks to resolve this infrastructure barrier and aims to accelerate translational research (10). Its main goal is "to identify major opportunities and gaps in biomedical research that no single institute at NIH could tackle alone." The roadmap covers three main themes:

- "New Pathways to Discovery": to stimulate the development of novel approaches to unravel the complexity of biologic systems and their regulation

- "Research Teams of the Future": to reduce the cultural and administrative barriers that often impede research and invoke an era in which scientists can cooperate in new and different ways

- "Re-engineering the Clinical Research Enterprise": to fund facilities, resources, or both to bolster clinical and translational research.

The Clinical and Translational Science Awards (CTSAs) were created as an outgrowth of the roadmap initiative. Interested applicants will propose transformative efforts appropriate to their own institutions (http:/grants.nih.gov/grants/ guide/notice-files/NOT-RM-05-013.html). The nature of these proposals should address educational programs, describe career pathway proposals for scientists investing in translational research, and provide a research environment that is flexible and both conducive to and responsive to the demands of modern translational and clinical research. The intention of the CTSA primary author, Dr. Zerhouni is that, "the CTSAs will advance the assembly of institutional academic homes providing integrated intellectual and physical resources for the conduct of original clinical and translational science $(11,12)$."

Specifically, the NIH has asked applicants to consolidate General Clinical Research Centers (GCRCs), T32 and K12 programs, and other resources as appropriate as they propose their individual integrated programs. Resources may be augmented by NIH Roadmap funding redirected from other initiatives and targeted to the CTSA program, with the National Center for Research Resources. The NIH consortium recently added 12 more academic health centers to the 12 announced last October (2006) - the funding through the CTSA program totaled $\$ 108$ million the first year. A third funding opportunity announcement for CTSAs has been issued, calling for the next round of applications to be submitted by November 7, 2007 with the awards expected in June 2008. This funding announcement and other information about the CTSA Program are available at www.ncrr.nih.gov/ctsa.asp. When fully implemented in 2012, 60 institutions will be linked together to invigorate the discipline of clinical and translational science (http://www.ctsaweb.org/). The awards are for 5 years and the program itself will eventually replace the GCRCs (13).

\section{THE UNIVERSITY OF PENNSYLVANIA/CHILDREN'S HOSPITAL OF PHILADELPHIA CTSA}

One of the initial 12 CTSA recipients was the University of Pennsylvania (Penn) and its partner institutions, the
Children's Hospital of Philadelphia (CHOP), the Wistar Institute and the University of the Sciences in Philadelphia (Pharmacy). Their application included participation from the Schools of Medicine, Nursing, Dentistry, Education, Arts and Sciences, Veterinary, Engineering and Applied Sciences, the Annenberg School of Communications, and the Wharton Business School within Penn combined with colleagues from external partnerships into novel, interdisciplinary structures and programs. Penn and $\mathrm{CHOP}$, together with their partner institutions include a large critical mass of senior faculty accomplished in the translation of a diverse array of therapeutic modalities-small molecules, proteins, genes, vaccinesinto the clinical domain. Together they have a remarkable training record-more NIH supported training grants than any other institution, the largest medical science training program in the country and multiple junior faculty holding $\mathrm{K}$ awards. They also have two well established NIH funded GCRCs with potential to bridge the pediatric/adult interface. Finally, the group represents a single geographic campus facilitating the engagement of chemists, engineers, statisticians, nurses, veterinarians, dentists, pharmacists, policy makers and shapers and experts in commercialization with biomedical scientists, epidemiologists and physicians.

The Penn/CHOP transformational plan involves a commitment by the institutions involved to (a) collaborate and support the recruitments and programs in the field of clinical and translational research; (b) devote substantial space (wet and dry laboratories) to clinical and translational research; and (c) foster the trans-institutional expansion of the "academic home" of this enterprise-the Institute for Translational Medicine and Therapeutics (ITMAT) - to permit development of new centers, cores and interdisciplinary programs of research and education (14). An important goal for the Penn/ CHOP plan is the development of focused strategic alliances with the FDA and the pharmaceutical and computing industries. Besides these alliances, the engagement of BioAdvance (http://www.bioadvance.com), a state funded entity charged with fostering the development of the life sciences in southeastern Pennsylvania has also been secured. BioAdvance will assist in providing resource for the access of trainees based with primary appointments in regional institutions to these educational instruments and will also support the development of and regionalization of access to bioinformatics platforms developed through the CTSA. Figure 1 shows the various centers which comprise ITMAT with the associated cores which will service the Translational Research Center. The support of quantitative pharmacology practice will be provided by the KMAS (Kinetic Modeling and Simulation) Core (http://www.med.upenn.edu/kmas/) which will (a) aid in the development of drug assays; (b) promote and assist in the performance of tracer kinetic studies; (c) develop novel approaches to kinetic data analysis; (d) provide PK, PK/PD, and tracer kinetic modeling; and (e) develop educational modules in pharmacokinetics and tracer kinetics to populate the educational initiatives pursued within the CTSA.

A central mission of ITMAT has been education. The deficiency of human capital in translational research, particularly with respect to the development and evaluation of new medicines is well appreciated and pervasive in industry, academia and the regulatory community (15). Figure 2 contains a schematic of the proposed educational program 


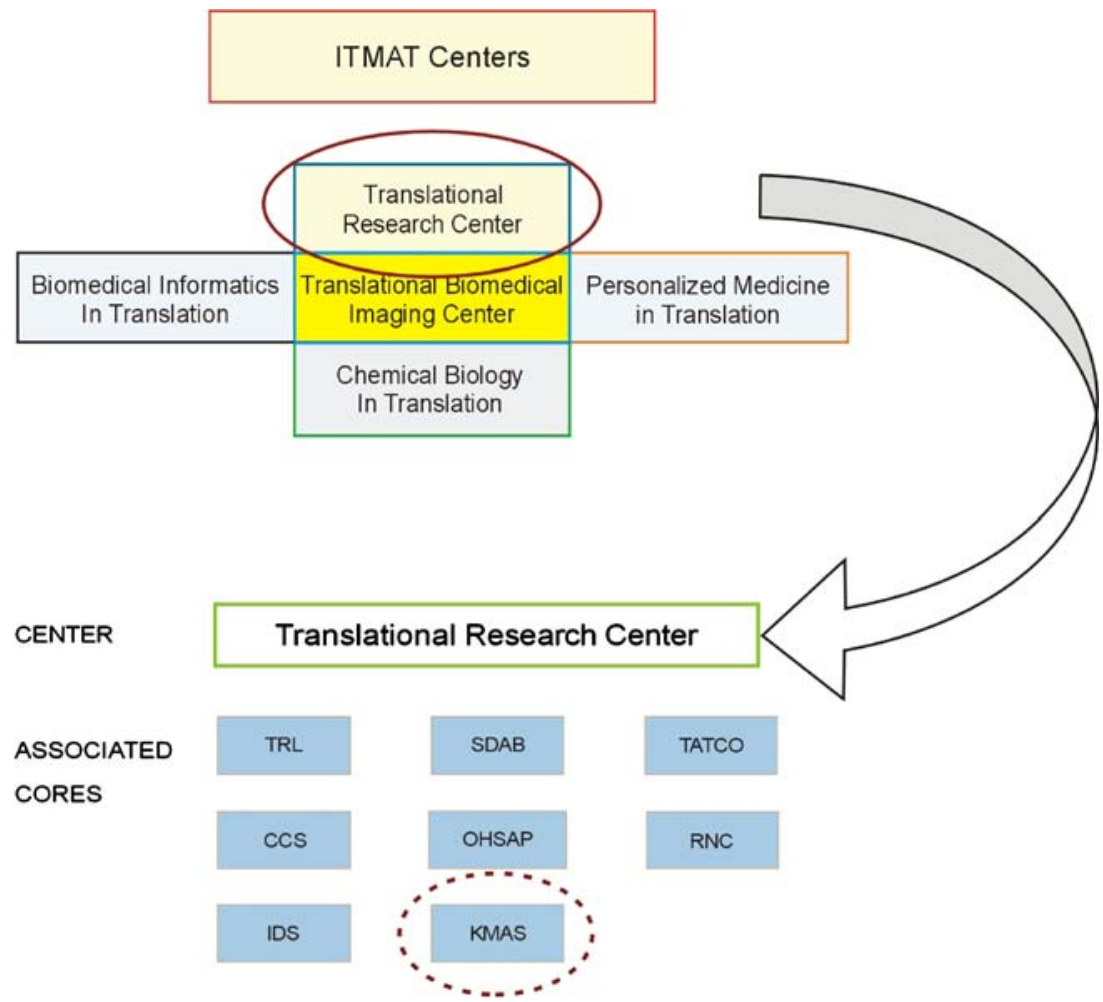

Fig. 1. Institute for Translational Medicine and Therapeutics (ITMAT) Centers proposed within the Penn/CHOP CTSA grant and the new cores supporting the Translational Research Center. The KMAS (Kinetic Modeling and Simulation) core will provide technologic expertise for integration of quantitative pharmacology into the translational research paradigm
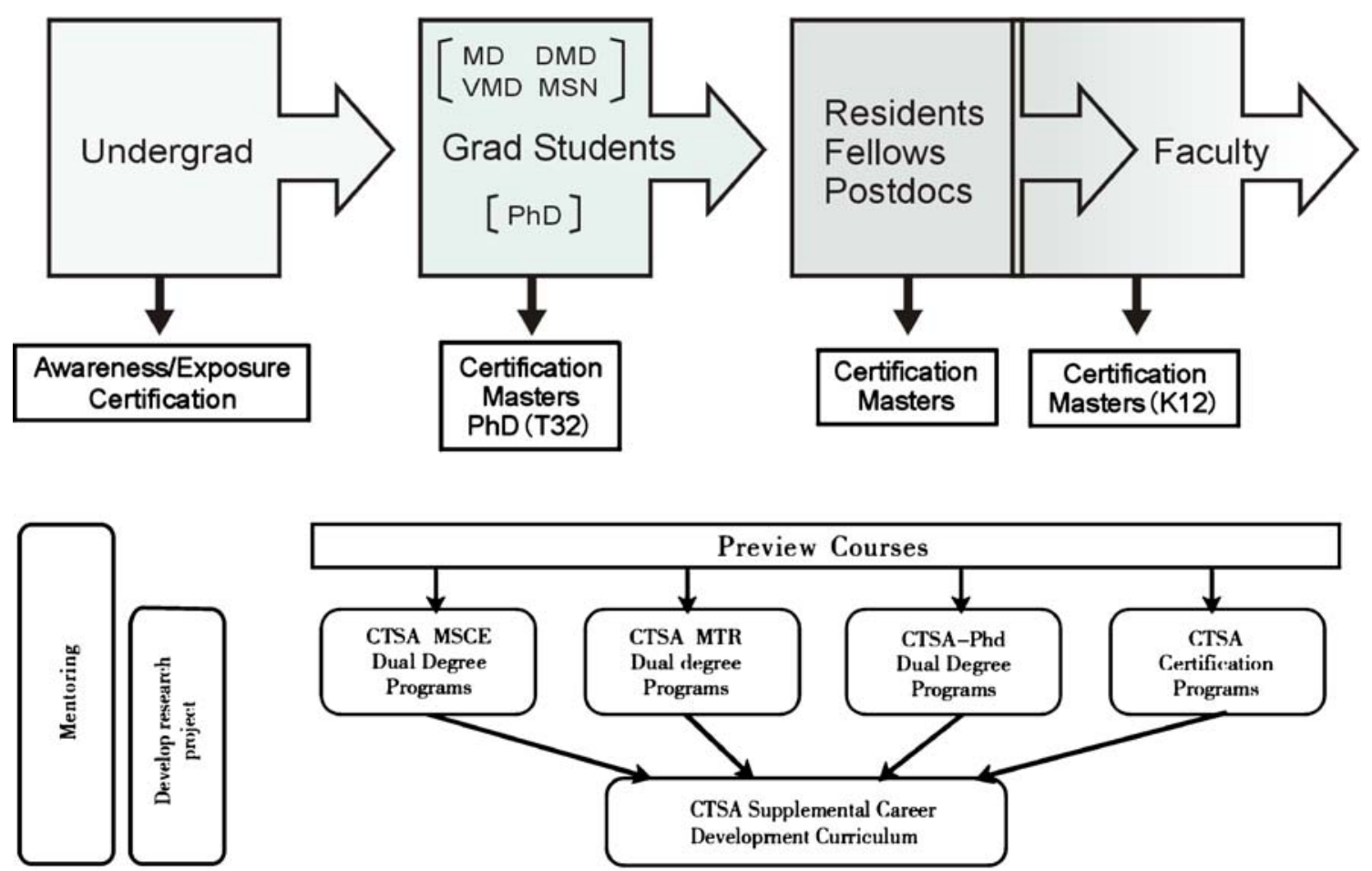

Fig. 2. The Penn/CHOP educational program proposed for the CTSA targets the full spectrum of potential and existing trainees, from undergraduates to graduate students, fellows, and faculty. As individuals require different levels and types of training at each stage of their academic development, multiple options are provided for exposure to translational research ranging from preview/awareness courses to certification, masters and doctoral degree programs 
supported by the Penn/CHOP CTSA. The ITMAT had proposed the name "Translational Medicine and Therapeutics" to embrace the projection of basic disciplines into the clinical domain with the objective of developing novel therapeutics. Such individuals would be trained in the development and projection of mechanism based biomarkers from cellular and model systems to guide rational dose selection. Furthermore, they would be trained to apply the emerging use and bioinformatic analysis of output from technologies, such as proteomics, metabolomics and genomics, to select between diverse molecules directed at a singular target. While the KMAS core will provide functional support of quantitative pharmacology activities, a Pharmaco- metric Training Unit will provide educational and training resources in addition to previously created programs in translational medicine. Initially, a module on tracer kinetics, pharmacokinetics, and compartmental and pharmacometric modeling will be offered as a core requirement in a Translational Therapeutics track and electively as a stand alone course or a component in other degree courses administered via ITMAT in support of the CTSA. PhRMA and FDA staff will participate, both as faculty participants and as sites for rotation site for CTSA students. In addition, scientists from the Metrum Institute (http://metruminstitute.org/about.html), a nonprofit organization dedicated to the advancement of quantitative modeling and simulation in biomedical research, with particu-

\section{Quantitation / M\&S Tasks}

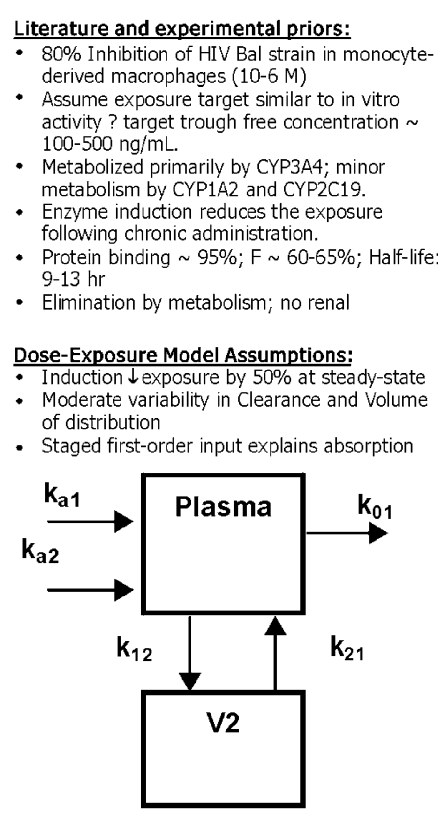

Simulation Model Results: interactions/adherence

- Induction may be manageable

- ARV/dose likely exceeds of CINV dose

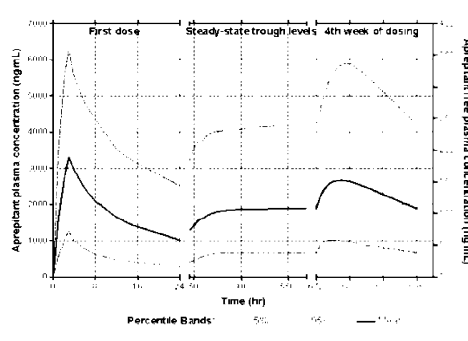

\section{Translational Workflow}

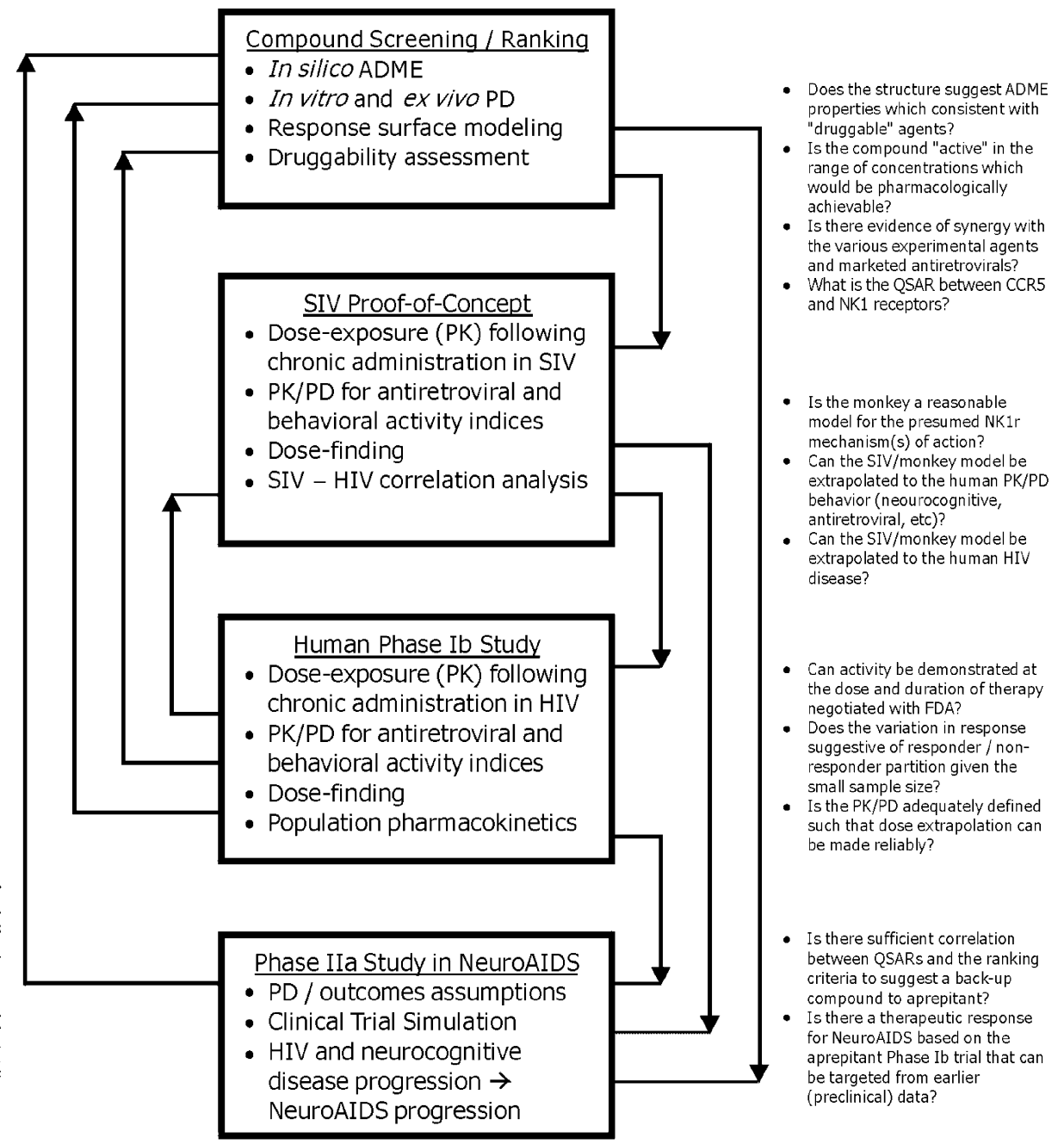

CINV $=$ chemotherapy-induced nausea and vomiting

SIV $=$ simian immunodeficiency virus

$\mathrm{ARV}=$ antiretroviral

Fig. 3. An example of quantitative pharmacologic principles applied to translational research: Relationships with aprepitant from in vitro data, in vivo data in animals, and in vivo data in CINV patients used to predict HIV-infected patient response and NeuroAIDS disease progression 
lar emphasis on problems in clinical pharmacology and therapeutics, will also serve as faculty. BioAdvance will facilitate regionalization of access to this program.

\section{CASE STUDY: NK1R ANTAGONISM IN NEUROAIDS}

An example of the integration of the quantitative pharmacology approach applied to an academic translational research initiative can be illustrated with recent efforts to explore NK1 receptor antagonism to treat NeuroAIDS. NeuroAIDS as a target indication is yet to be recognized as a separate therapeutic area by PhRMA companies though neurological complications associated with HIV-1/AIDS are being recognized with a frequency that parallels the increased number of AIDS cases. The early infiltration by HIV-1 into the nervous system can cause primary and/or secondary neurological complications. Likewise, these complications have been shown to ultimately influence patient compliance with their antiretroviral therapy. The National Institutes of Allergy and Infectious Disease and the National Institutes of Mental Health of the NIH (PAR-03-138) have funded the establishment of a multidisciplinary research and development program targeted toward the discovery, development and evaluation of innovative therapies for HIV infection (http://grants.nih.gov/grants/guide/pa-files/PAR-03-138.html). The overall goal of the program is to support and accelerate scientific and technical progress in non-traditional and traditional drug-based therapies that exploit novel viral and cellular targets of importance in HIV infection. A benchmark for a successful program is the development of a new treatment concept that can be introduced to clinical practice. The ability of neurokinin-1 receptor (NK-1R) antagonists to target the substance $\mathrm{P}$ receptor demonstrating antiviral and immunomodulatory effects (16-18) represents a new therapeutic target with the potential to interrupt a pathway critical to HIV replication $(19,20)$. The goal of this specific Integrated Preclinical/Clinical Program (IPCP) is to identify an NK-1R antagonist that is: (a) active as an anti-HIV agent through interaction with chemokine/cytokine receptors (Project 1); (b) specific for chemokine and G-protein coupled receptors (Project 2); (c) safe for use in SIV (simian immunodeficiency virus)-infected non-human primates and provides proof of concept related to antiviral, immunomodulatory, and neurobehavioral effects (Project 3); and (d) safe in humans and has positive immunomodulatory effects (Project 4). All projects contribute to understanding the basic virologic, molecular and cellular immunologic mechanisms of Substance P, NK-1R antagonists, and HIV/SIV infection.

A key element in the IPCP is the integration of modeling and simulation strategies to support the various projects as well as inform the administrative core which is ultimately responsible for project and overall grant decision making (go/ no-go decisions, candidate selection, etc). Specifically, computational techniques are employed to challenge the "druggability" of the candidate agents. In silico ADME (absorption, distribution, metabolism and elimination) techniques are used as part of the ranking criteria by which we will prioritize the advancement of selected agents. The approach enables the calculation of molecular descriptors and prediction of druglikeness data using 2D molecular structures, and without the need for special computational chemistry knowledge. As pharmacology and biology/mechanism data are generated for the various agents (Projects 1 and 2), the generalizability of structure-activity and structure-pharmacokinetic relationships for this compound class will be explored. Figure 3 illustrates the integration of quantitative pharmacology application across projects for this IPCP.

The NK1R project is dependent on two primary progression pathways: 1) examine the extent to which the NK1R antagonist aprepitant is a suitable agent for antiretroviral therapy in NeuroAIDS patients based on exposure-response criteria constructed from its presumed actions, and 2) examine the correlation between aprepitant preclinical pharmacology, druggability criteria and PK/PD relationships to generalize and ultimately rank suitable back-up compounds. Within the first pathway there is a progression of experiments, predefined criteria for stage advancement and decision trees that guide the overall progression. These are facilitated by modeling and simulation exercises permitting scenario testing for subsequent experiments and testing of assumptions fundamental to key decision criteria for the overall program. One of the essential bridges in this IPCP is the suitability of an SIV pharmacology/disease model to predict success outcomes in HIV. In the series of experiments that has ensued, the PK/PD of aprepitant in SIV-infected animals have been characterized and used in conjunction with relevant data from chemotherapyinduced nausea and vomiting patients to build simulation models that project response in HIV-infected patients. The use of modeling and simulation to advance compound progression of antiretroviral agents recently reviewed by Barrett (21) contains a more thorough description of this case study.

\section{CONCLUSION}

It is clear that academic medical centers will be an important player in the evolving R\&D paradigms which will guide the discovery of new molecular entities in the years to come. Translational research is an approach that relies heavily on the interaction between basic and clinical scientists and the appropriate communication of ideas, hypotheses and experimental designs to evaluate disease therapies. The concept of quantitative pharmacology is, in essence, a communication platform by which translational research can be discussed and in which assumptions, models and simulations are integrated into a decision-making dialogue. It is also clear that the recent efforts of the FDA and NIH reflect the recognition that this skillset is in short supply and will require dedicated programs to train future generations of scientists that conduct translational research.

\section{ACKNOWLEDGEMENTS}

Dr. Barrett's efforts are supported by NIH Grant's, P01 \# MH076388 and 1U54 \# RR023567-01.

\section{REFERENCES}

1. A. K. Rustgi. Translational research: what is it? Gastroenterology 116(6):1285 (1999).

2. R. Rosell, M. Monzo, A. O'Brate, and M.Taron. Translational oncogenomics: toward rational therapeutic decision-making. Curr. Opin. Oncol. 14(2):171-179 (2002). 
3. G. J. Kelloff, C. C. Sigman, and P. Greenwald. Cancer chemoprevention: progress and promise. Eur. J. Cancer. 35(14):2031$2038(1999)$

4. H. D. Humes. Translational medicine and the National Institutes of Health road map: steep grades and tortuous curves. J. Lab. Clin. Med. 146(2):51-54 (2005).

5. J. Mao. Translational pain research: bridging the gap between basic and clinical research. Pain 97(3):183-187 (2002).

6. S. C. Brown, and D. C. Park. Theoretical models of cognitive aging and implications for translational research in medicine. Gerontologist 43(Spec No 1):57-67 (2003).

7. CDER. Innovation and stagnation: Challenge and opportunity on the critical path to new medicinal products. US Food and Drug Administration (2004).

8. E. Zerhouni. Medicine The NIH Roadmap. Science 302(5642): 63-72 (2003).

9. J. Loscalzo. The NIH budget and the future of biomedical research. N. Engl. J. Med. 354(16):1665-1667 (2006).

10. H. Horig, E. Marincola, and F. M. Marincola. Obstacles and opportunities in translational research. Nat. Med. 11(7):705-708 (2005)

11. E. A. Zerhouni. Translational and clinical science-time for a new vision. N. Engl. J. Med. 353(15):1621-1623 (2005).

12. E. A. Zerhouni, and B. Alving. Clinical and translational science awards: a framework for a national research agenda. Translation Research 148(1):4-5 (2006).

13. J. Kaiser. Biomedicine. NIH funds a dozen 'homes' for translational research. Science 314(5797):237 (2006).
14. K. Garber. Translational institute unites unlikely partners at Penn. Science 317:968-969 (2007)

15. D. Dauphinee, and J. B. Martin. Breaking down the walls: thoughts on the scholarship of integration. Acad Med 75(9):881$886(2000)$

16. W. Z. Ho, A. Cnaan, Y. H. Li, H. Zhao, H. R. Lee, L. Song, and S. D. Douglas. Substance P modulates human immunodeficiency virus replication in human peripheral blood monocyte-derived macrophages. AIDS Res. Hum. Retrovir 12:195-198 (1996).

17. W. Z. Ho, J. P. Lai, Y. Li, and S. D. Douglas. HIV enhances substance $\mathrm{P}$ expression in human immune cells. FASEB $J$. 16:616-618 (2002).

18. J. P. Lai, W. Z. Ho, G. X. Zhan, Y. Yi, R. G. Collman, and S. D. Douglas. Substance P antagonist (CP-96,345) inhibits HIV-1 replication in human mononuclear phagocytes. Proc. Natl. Acad. Sci. U. S. A. 98:3970-3975 (2001).

19. S. D. Douglas, W. Z. Ho, D. R. Gettes, A. Cnaan, H. Zhao, J. Leserman, J. M. Petitto, R. N. Golden, and D. L. Evans. Elevated substance $\mathrm{P}$ levels in HIV-infected men. AIDS 15 2043-2045 (2001).

20. D. L. Evans, J. Leserman, D. O. Perkins, R. A. Stern, C. Murphy, K. Tamul, D. Liao, C. M. Horstvan der, C. D. Hall, and J. D. Folds. Stress-associated reductions of cytotoxic T lymphocytes and natural killer cells in asymptomatic HIV infection. Am. J. Psychiatry 152:543-550 (1995).

21. J. S. Barrett. Facilitating compound progression of antiretroviral agents via modeling and simulation. J. Neuroimmune Pharmacol 2:58-71 (2007). 